\title{
Diapause Induction In Cotesia Plutellae: Who's More Caring About Their Progeny Fate Mothers Or Fathers And What Is The Maternal Stage Taking The Responsibility For Diapause Induction in the Progeny
}

\author{
Umsalama Abdelmageed Ahmed ${ }^{1}{ }^{2} \mathrm{SHI} \mathrm{Zu}$-hua \\ ${ }^{I}$ Department of Biology, Faculty of Science and Art, Taif University, khurma Branch, Khurma, Kingdom of Saudi \\ Arabia \\ ${ }^{2}$ Institute of Insect Sciences, Zhejiang University, Hangzhou 310029, China
}

\begin{abstract}
These novel results indicate that embryos can respond directly to the environmental conditions they experience during development, but that their ability to do so is influenced by maternal effects such as parental diapause history. The results of this study not only provide evidence, for the first time, of parental diapause history affecting diapause proportions, but also raise additional questions about the mechanism by which environmental information is transmitted from parent to offspring and how offspring are able to respond to conditions experienced during their own development.
\end{abstract}

Keywords: diapause, photoperiod, mother, sensitive stage

\section{Introduction}

Most insect species have evolved a period of developmental hold (diapause) that enables them to avoid seasonal periods of difficulty. For insects in the temperate zone, winter is the season most consistently avoided. Many insect species rely on environmental factors such as photoperiod, temperature and host availability as a cause for the induction of diapause. In some species, diapause is influenced by the environmental conditions experienced by a parental insect. Mousseau and Fox (1998) highlighted the idea that mothers transmit information about environmental variability to their offspring and proposed that these maternal affects are a mechanism for adaptive phenotypic response to changing environments. Moreover environmentally induced maternal effects have been documented in many insect species (Mousseau and Fox 1998). Maternal effects occur when a female alters developmental characteristics of her offspring, often in response to some aspect of the environment she has experienced during her lifetime (Mousseau and Dingle 1991; Mousseau and Fox 1998). Females that experience short-day conditions, low temperature, or a scarcity of potential hosts, tend to produce a high proportion of diapausing offspring (Mousseau and Fox, 1998).

The occasion of diapause in Cotesia plutellae was studied by Alvi and Momi, 1994 who found that C. plutellae enter diapause in a mature third larval instar (prepupa) and its diapause was influenced by the photoperiodic conditions experienced by the larvae themselves (Alvi and Momi, 1994) since they were not studied the parental effect of diapause induction in this parasitoid.

According to our knowledge, the effects of parental photoperiodic history on progeny critical photoperiod for diapause induction have not been studied before. As maternal photoperiod history is usually examined by testing the effect of different maternal photoperiod on diapause induction in the progeny. Therefore, we tested the influences of parental photoperiod history on critical photoperiod for diapause incidence in the progeny.

We designed our study to test different specific hypothesis on diapause induction in C. plutellae came from south China'.

1) Does of parental photoperiodic history affect the progeny critical photoperiod for diapause induction in $C$. plutellae?

2) Does the parental critical photoperiod affected by the progeny photoperiod in C. plutellae?

3) Does the different parental developmental stage condition affect diapause induction in the progeny of $C$. plutellae?

4) Does the diapause induction in C. plutellae under mother control? 
Diapause Induction In Cotesia Plutellae: Who's More Caring About Their Progeny Fate Mothers ..

Insect cultures and host plants

\section{Material and methods}

A laboratory culture of Cotesia plutellae was established from $\mathrm{ca} .100$ cocoons collected in September 2003 on Brassica plants at suburbs of Changchun, Jilin province, China ( $\left.43^{\circ} 52^{\prime} \mathrm{N}\right)$. The wasps were maintained continuously in the laboratory on a potted cabbage with third and fourth instar larvae of $P$. xylostella as a host. $P$. xylostella was reared on a potted cabbage, Brassica oleracea var., capitata, cv. Jingfeng NO 1, according to the method described by Wang et al. (1999). Both the parasitoid and host insect cultures were reared in temperature-controlled rooms at temperatures between 25 and $30^{\circ} \mathrm{C}$, relative humidity of $60-80 \%(\mathrm{RH})$ with LD 12:12 h. The stock culture of the parasitoid had been reared for 8-10 generations since it was establishment in our laboratory.

\section{Experimental insects}

To obtain host larvae parasitized by $C$. plutellae, DBM larvae were exposed one at a time to female of $C$. plutellae in $18 \mathrm{~mm} \times 80 \mathrm{~mm}$ glass tube and the insects were monitored until oviposition by a wasp was seen, then $P$. xylostella larvae were collected and transferred directly to rearing cages. Thirty parasitized DBM larvae were placed on a cabbage leaf held in a plastic container. The container was made of a clear plastic cup (110 mm in diameter, $110 \mathrm{~mm}$ in height) with holes covered with a fine stainless steel mesh on its top and bilateral walls for ventilation. The cabbage leaf carrying host larvae was fixed to the container base by inserting its petiole through a hole in the base center. The container was placed on top of a 500-ml glass bottle with tap water, and the petiole of the cabbage leaf was kept immersed in water to maintain freshness. The cabbage leaf was replaced when need.

\section{Determination of diapausing individuals}

Based on the relationship between developmental rate $(y)$ and temperature $(x)(y=-0.1909+0.0163 x)$, the period of development of non-diapause parasitoid stage inside the cocoon was 24 days at $15^{\circ} \mathrm{C}$ (Shi and Liu, 1999). Parasitoid cocoons, from which no wasp had emerged after aforementioned period were dissected under stereomicroscope. The dissected cocoons were classified into two categories: non-diapause (containing dead larva, dead prepupa, pupa or adult) or diapause (containing live larvae or prepupa). The diapause incidence was calculated using the following formula.

$\%$ Diapause $=($ number of cocoons with live larvae or with live prepupa / total cocoon number collected cocoon number with dead larvae) $\times 100$.

\section{Effect of parental photoperiod on critical day length for diapause induction}

To examine the effect of parental photoperiod on critical day length, maternal generation was reared from egg until adult emergence at three different photoperiods (LD 8:16, 10:14, 12:12, 14:10, 16:8 h). Emerged adults from each treatment were used to parasitize the host larvae as described previously. Parasitized host larvae were reared at $15^{\circ} \mathrm{C}$ and five different photoperiods (LD 8:16, 9:15, 10:14, 11:13, 12:12, 13:11, 14:10h). Five replicates were used and each replicate contained thirty parasitized host larvae. The collected cocoons were remained as their prior developmental stages and allowed either to enter diapause or developed to adult.

\section{Effect of each parent in diapause incidence}

In $C$. plutellae, the induction of larval diapause is influenced by photoperiod and temperature experienced by the larvae themselves and by photoperiod experienced by their parents (Ahmed et al., 2006). To examine which of the parents who determines the diapause in the progeny, groups of three rearing cages (per each treatment) represented insects of the parental generation were kept in programmable incubators set at $25^{\circ} \mathrm{C}$ with LD 8:16h, LD12:12h or LD16:8.h. Collected cocoons from each treatments were kept separately (one cocoon in a separate test tube). Emerged adults from each treatment were collected, sexed and housed in a small single sex cages until sufficient had been obtained for the crosses. Cotton ball containing $20 \%$ honey solution was added to each cage as a food source for parasitoid wasps. Cages of nine combinations of adult were set up as shown in table 1. Two days later one pair of female and male (from each treatment) were transferred into separate test tubes and then allowed to parasitize the host larvae as described earlier. Twenty female were used to parasitize the host larvae for each treatments. Parasitized host larvae from each cross were reared under $15^{\circ} \mathrm{C}$ LD 11:13 h until cocoon formation. The collected cocoons were kept as their larval conditions and were checked daily to asses adult emergence.

\section{Determination of the maternal sensitive stage}

According to this recent study in $C$. plutellae the mother is determine the fate of her progeny whether entered diapause or not. Furthermore when larvae of this parasitoid are reared under $15^{\circ} \mathrm{C}$ LD $11: 13 \mathrm{~h}$ the incidence of diapause depends on the parental photoperiod: $21 \%$ of the larvae of parents exposed to LD 16:8 at 
Diapause Induction In Cotesia Plutellae: Who's More Caring About Their Progeny Fate Mothers ..

$25^{\circ} \mathrm{C}$ enter diapause, whereas $80 \%$ larvae of parents exposed to LD $12: 12$ at $25^{\circ} \mathrm{C}$ do (Ahmed et al., submitted 2006). In the present study, the paternal generation was reared at $25^{\circ} \mathrm{C} \mathrm{LD} 16: 8 \mathrm{~h}$ (since the father had no fate on his progeny development), whereas the maternal generation was exposed to different combinations of LD 12: 12 and $16: 8 \mathrm{~h}$ at $25^{\circ} \mathrm{C}$ in the larval, pupal and adult stages. Emerged adults were allowed to parasitized host larvae as described previously. The parasitized host larvae were reared under constant temperature $15^{\circ} \mathrm{C}$ and LD 11:13. Five replicates were used per each treatment and each replicate contained thirty parasitized host larvae. Collected cocoons remained in the same conditions $\left(15^{\circ} \mathrm{C}\right.$ and LD 11:13 $\left.\mathrm{h}\right)$ as their immature stages and allowed either to develop to adult or entered diapause.

\section{Statistical analysis}

Following arcsine square root-transformation on frequency data, the effect of parental photoperiod and diapause induction was analyzed using two-way analysis of variance ANOVA whereas parental sensitive stage for diapause induction was analyzed using one-way analysis of variance ANOVA after. Significant different was detected means were separated by Duncan's Comparison Test using the SAS statistical software package (SAS institute, 2000. v.8.1).

\section{Results}

Effect of parental photoperiod on diapause induction

All larvae produced by the parents kept at $25^{\circ} \mathrm{C}$ and LD 8, 10, $12 \mathrm{~h}$ light and reared under $15^{\circ} \mathrm{C}$ with LD 8, 9, 10 enter diapause, whereas the percentage of the larvae entered diapause reared as the same condition $\left(15^{\circ} \mathrm{C}\right.$ with $\left.\mathrm{LD} 8,9,10\right)$ was sharply affected when the day length under which the parental generation was kept became longer than $12 \mathrm{~h}$ of light per day (Table 1). The results showed that the induction of diapause depended on both parental and progeny photoperiod. Table 1 shows that all parental photoperiods $\leq 12 \mathrm{~h}$ of light per day resulted in high incidence of diapause $(>50 \%)$ when combined with the progeny photoperiod $\leq 11 \mathrm{~h}$ of light per day. The critical photoperiod for diapause induction in progeny produced by parent kept at $\leq 12 \mathrm{~h}$ of light per day was fall between 11 and $12 \mathrm{~h}$ light where as in the progeny produced by parent kept at LD 12:12 $\mathrm{h}$ the critical photoperiod was fall between 9-10 h light (Table 1). The parental kept at LD 16:8 h produced lower than $50 \%$ diapause in their progeny at all examined photoperiod (Table 1). Also as the photoperiod under which the larvae were kept was prolonged, diapause incidence in the progeny was significantly influenced by the parental photoperiod (Table 2). Again the parental critical photoperiod for diapause induction was affected by the photoperiod under which the progeny was reared. The parental critical photoperiod was longer at the shorter photoperiod under which the progeny was reared. For example when we reared the progeny at LD 8:16, 9:15 the parental critical photoperiod was fall between 14-16 h light per day, whereas in progeny reared at LD10:14 and 11:12 $\mathrm{h}$ the parental critical photoperiod was fall between 12-14 h light per day light (Table 3).

Table (1) Diapause incidence in larvae of Cotesia plutellae produced by parents that experienced different photoperiods at $25^{\circ} \mathrm{C}$ The larvae produced by these parents were reared under different photoperiods at $15^{\circ} \mathrm{C}$.

\begin{tabular}{|c|c|c|}
\hline Mother generation photoperiod (L: D h) & Progeny generation photoperiod (L: D h) & Progeny Diapause (\%) \\
\hline \multirow[t]{7}{*}{$8: 16 \mathrm{~A}$} & $8: 16$ & $100.00 \pm 0.00 \mathrm{~A}$ \\
\hline & $9: 15$ & $100.00 \pm 0.00 \mathrm{~A}$ \\
\hline & $10: 14$ & $100.00 \pm 0.00 \mathrm{~A}$ \\
\hline & $11: 13$ & $97.12 \pm 1.33 \mathrm{~B}$ \\
\hline & $12: 12$ & $45.26 \pm 2.54 \mathrm{C}$ \\
\hline & $13: 11$ & $38.07 \pm 1.39 \mathrm{D}$ \\
\hline & $14: 10$ & $34.69 \pm 2.24 \mathrm{E}$ \\
\hline \multirow[t]{7}{*}{$10: 14 \mathrm{~A}$} & $8: 16$ & $100.00 \pm 0.00 \mathrm{~A}$ \\
\hline & $9: 15$ & $100.00 \pm 0.00 \mathrm{~A}$ \\
\hline & $10: 14$ & $100.00 \pm 0.00 \mathrm{~A}$ \\
\hline & $11: 13$ & $98.00 \pm 0.49 \mathrm{~B}$ \\
\hline & $12: 12$ & $43.91 \pm 2.33 \mathrm{C}$ \\
\hline & 13:11 & $31.17 \pm 1.54 \mathrm{D}$ \\
\hline & $14: 10$ & $30.45 \pm 1.57 \mathrm{D}$ \\
\hline \multirow[t]{7}{*}{$12: 12 \mathrm{~A}$} & $8: 16$ & $100.00 \pm 0.00 \mathrm{~A}$ \\
\hline & $9: 15$ & $100.00 \pm 0.00 \mathrm{~A}$ \\
\hline & $10: 14$ & $100.00 \pm 0.00 \mathrm{~A}$ \\
\hline & $11: 13$ & $80.31 \pm 3.58 \mathrm{~B}$ \\
\hline & $12: 12$ & $41.58 \pm 1.16 \mathrm{C}$ \\
\hline & $13: 11$ & $31.74 \pm 2.05 \mathrm{D}$ \\
\hline & $14: 10$ & $30.63 \pm 0.49 \mathrm{D}$ \\
\hline \multirow[t]{4}{*}{ 14:10B } & $8: 16$ & $78.10 \pm 0.08 \mathrm{~A}$ \\
\hline & $9: 15$ & $60.90 \pm 4.34 \mathrm{~A}$ \\
\hline & $10: 14$ & $30.00 \pm 7.28 \mathrm{~B}$ \\
\hline & $11: 13$ & $27.30 \pm 5.45 \mathrm{~B}$ \\
\hline
\end{tabular}


Diapause Induction In Cotesia Plutellae: Who's More Caring About Their Progeny Fate Mothers ..

\begin{tabular}{|l|l|l|}
\hline & $12: 12$ & $14.64 \pm 2.27 \mathrm{C}$ \\
\hline & $13: 11$ & $4.48 \pm 2.41 \mathrm{C}$ \\
\hline $16: 8 \mathrm{C}$ & $14: 10$ & $0.00 \pm 0.00 \mathrm{E}$ \\
\hline & $8: 16$ & $27.60 \pm 7.05 \mathrm{~A}$ \\
\hline & $9: 15$ & $22.90 \pm 5.07 \mathrm{~A}$ \\
\hline & $10: 14$ & $25.50 \pm 4.76 \mathrm{~A}$ \\
\hline & $11: 13$ & $21.60 \pm 4.62 \mathrm{~A}$ \\
\hline & $12: 12$ & $6.40 \pm 1.20 \mathrm{~B}$ \\
\hline & $13: 11$ & $0.00 \pm 0.00 \mathrm{C}$ \\
\hline
\end{tabular}

Means \pm SE followed by the same letters with in each mother photoperiod are not significantly different according to Duncan's comparison of mean.

Table (2) Two way analysis of variance testing the effect of parental and progeny photoperiod on diapause induction of Cotesia plutellae.

\begin{tabular}{|l|l|l|l|}
\hline \multicolumn{1}{|c|}{ Source } & \multicolumn{1}{c|}{ DF } & \multicolumn{1}{c|}{ MS } & \multicolumn{1}{c|}{ F value } \\
\hline Mother photpperiod (A) & 4 & 11568.46 & $246.55^{* * *}$ \\
\hline Progeny photoperiod (B) & 6 & 7241.31 & $145.33^{* * *}$ \\
\hline A $\times$ B & 24 & 194.94 & $145.33^{* * *}$ \\
\hline
\end{tabular}

Table (3) Parental critical photoperiod for diapause induction in Cotesia plutellae. parents was reared at $25^{\circ} \mathrm{C}$ and the produced progeny were kept under $15^{\circ} \mathrm{C}$.

\begin{tabular}{|c|c|}
\hline Parental critical photoperiod & Progeny photoperiod (LD h) \\
\hline 14-16 h light & $8: 16 \mathrm{~h}$ \\
\hline 14-16 h light & $9: 15 \mathrm{~h}$ \\
\hline 12-14 h light & $10: 14 \mathrm{~h}$ \\
\hline 12-14 h light & $11: 13 \mathrm{~h}$ \\
\hline
\end{tabular}

Effect of each parent in diapause incidence (reciprocal crosses)

Almost all the larvae produced by mothers reared under LD 8:16 $\mathrm{h}$ at $25^{\circ} \mathrm{C}$ enter diapause, whether the fathers experienced LD 8:16, 12:12 or $16: 8 \mathrm{~h}$ at $25^{\circ} \mathrm{C}$ (Table 4). Also, a greater proportion of larvae produced by mothers kept under LD 12:12 at $25^{\circ} \mathrm{C}$ entered diapause (Table 4). When the mother kept at LD 16:8 h a greater percentage of the progeny averted diapause irrespective to the conditions under which the father was kept (Table 4). The incidence of diapause in progeny was significantly influenced by the conditions experienced by mothers, but not by the conditions experienced by the fathers.

Table (4) the larval diapause percentage in each combination of maternal and parental photoperiods

\begin{tabular}{|l|l|}
\hline $\begin{array}{l}\text { Crosses } \\
(\times+)\end{array}$ & Diapause incidence \\
\hline $8 \mathrm{~h} \mathrm{~L} \times 8 \mathrm{~h} \mathrm{~L}$ & $97.12 \pm 1.33 \mathrm{~A}$ \\
\hline $8 \mathrm{~h} \mathrm{~L} \times 12 \mathrm{~h} \mathrm{~L}$ & $95.26 \pm 1.52 \mathrm{~A}$ \\
\hline $8 \mathrm{~h} \mathrm{~L} \times 16 \mathrm{~h} \mathrm{~L}$ & $98.62 \pm 1.38 \mathrm{~A}$ \\
\hline $12 \mathrm{~h} \mathrm{~L} \times 8 \mathrm{~h} \mathrm{~L}$ & $78.75 \pm 4.42 \mathrm{~B}$ \\
\hline $12 \mathrm{~h} \mathrm{~L} \times 12 \mathrm{~h} \mathrm{~L}$ & $80.31 \pm 3.58 \mathrm{~B}$ \\
\hline $12 \mathrm{~h} \mathrm{~L} \times 16 \mathrm{~h} \mathrm{~L}$ & $77.25 \pm 1.54 \mathrm{~B}$ \\
\hline $16 \mathrm{~h} \mathrm{~L} \times 8 \mathrm{~h} \mathrm{~L}$ & $21.73 \pm 1.9 \mathrm{C}$ \\
\hline $16 \mathrm{~h} \mathrm{~L} \times 12 \mathrm{~h} \mathrm{~L}$, & $26.45 \pm 3.72 \mathrm{C}$ \\
\hline $16 \mathrm{~h} \mathrm{~L} \times 16 \mathrm{~h} \mathrm{~L}$ & $21.6 \pm 4.62 \mathrm{C}$ \\
\hline
\end{tabular}

Means \pm SE flowed by the same letter with in each maternal photoperiod are not significantly different according to Duncan Multiple Range Analysis Test.

\section{Maternal sensitive stage for diapause induction}

Conditions experienced by the parents in $C$. plutellae during their immature stages appear to affect the diapause incidence in the progeny. Females from two stages exposed to short photoperiod produced high diapause incidence in the progeny. Egg and cocoon stage or cocoon and adult stages together exposed to short day condition produce statistically same diapause incidence 76.92 and $75.12 \%$ respectively (Table 5). Parental adult stage also seemed to be sensitive to diapause induction. Adult stage exposed to short day condition (Ttreatment 2 and 5) produced 53.19 and $56.67 \%$ diapausing individuals. When parasitoids were kept under long photoperiod in their cocoon and adult stage together the incidence of diapause is very low and statistically not different from that of parasitoid kept under short photoperiod only at the cocoon stage. Additional treatment was carried out to confirm the effect of the adult on diapause incidence through which we exposing the cocoon 
Diapause Induction In Cotesia Plutellae: Who's More Caring About Their Progeny Fate Mothers ..

stage to a shorter photoperiod (see table 5 treatment 7) the diapause incidence was statistically the same as when we exposed the adult to shorter photoperiod (treatments 2 and 5).

Table (5) Diapause incidence in larvae of Cotesia plutellae observed with different combinations of parental conditions in the egg-larval, cocoon and adult stages. The larvae produced by these parents were reared under Ld $11: 13 \mathrm{~h}$ at $15^{\circ} \mathrm{C}$

\begin{tabular}{|l|l|l|l|l|}
\hline Treatment no & Egg-larval & Cocoon & Adult & \\
\hline 1 & S & S & L & $76.92 \pm 2.2 \mathrm{~A}$ \\
\hline 2 & L & L & S & $53.19 \pm 2.59 \mathrm{~B}$ \\
\hline 3 & L & S & S & $75.12 \pm 2.84 \mathrm{~A}$ \\
\hline 4 & S & L & L & $35.14 \pm 3.15 \mathrm{C}$ \\
\hline 5 & S & L & S & $56.67 \pm 2.53 \mathrm{~B}$ \\
\hline 6 & L & S & L & $41.18 \pm 2.43 \mathrm{C}$ \\
\hline 7 & L & S* & L & $56.36 \pm 3.19 \mathrm{~B}$ \\
\hline
\end{tabular}

Means \pm SE flowed by the same letter with in the same column are not significantly different according to Duncan Multiple Range Analysis Test.

\section{Effect of parental photoperiod on diapause induction}

\section{Discussion}

Maternal effects are well known to manipulate offspring performance (e.g. Leimar and McNamara 2015; Saunders 2010; Ho and Burggren 2010). The idea that mothers transmit information about environmental variability to their offspring is well addressed by Mousseasu and Fox (1998) and they proposed that these maternal effects are a mechanism for adaptive phenotypic response to changing environment. In Univoltine and bivoltine insects, decreasing photoperiods and temperatures can acts as cues for mothers to produce diapausing offspring (Mousseau and Dingle 1991). In the present study we found in C. plutellae there is direct effect of parental photoperiod on the influence of diapause in the offspring (Table 1). Parents whose experienced short photoperiod produced more diapause in their progeny our result is consistence with the result obtained by Tachibana and Numata, 2004 they were reported that in blow fly Lucilia sericata the diapause in the progeny was highly affected by the temperature and photoperiods experienced by parental generation. In our result we found there is significant interaction between critical photoperiod for diapause induction in both parents and progeny. Since the critical photoperiod for diapause induction in the progeny produce by the parents experienced short period was longer than in the progeny whose parents experienced longer photoperiod. This is refereed to the fact that short photoperiod in parental condition produce high diapause in the progeny.

Parental critical photoperiod for diapause induction in $C$. plutellae was greatly affected by the photoperiod experienced by the progeny so as the photoperiod under which the progeny was kept increased the critical parental photoperiod was decreased.

It is reasonable to assume that each female has a threshold daylength value enabling her to distinguish long from short days. As day length decrease, more and more female are below their own threshold and the level of diapause amongst the offspring of the population increases. The reverse happens as day lengths increase (MCwatters and Saunders, 1996). Reznik et al. 2011a in their strictest hypothesis described that mothers experienced environmental cue and produce offspring appropriate for the conditions that the progeny are expected to encounter. These parental effects should be favored by natural selection because they have an adaptive value, enhancing the probability of survival and reproduction of progeny.

\section{Maternal sensitive stage for diapause induction}

In $C$. plutellae, the present results with $\mathrm{LD} 16: 8$ and $\mathrm{LD} 12: 12 \mathrm{~h}$ at $25^{\circ} \mathrm{C}$ show that cocoons experienced short photoperiod when followed by egg-larval or adults stage are the key stage for parental induction of diapause, though the adult stage is also sensitive but its not much as the cocoon stage because when we exposed cocoon stage to $8 \mathrm{~h}$ of light per day the diapause percentage is statistically same as when exposed the adult stage to $12 \mathrm{~h}$ of light per day. There are a small number of insects in which parental stages other than the adult are reported to be sensitive for induction of larval diapause (Anderson and Kaya 1974; Milonas and Savopoulou-Soultani 2000).

\section{Effect of each parent in diapause incidence (reciprocal crosses)}

In most insects (Ho and Burggren, 2010, Jason. P et al 2010, Yamaguchi and Nakamurak 2015) parental influence on diapause induction has been studied, it has been believed that diapause of progeny is regulated maternally, without examining whether the mother or the father influence the expression of diapause in the progeny. Although diapause induction in C. plutellae was under parental control (Ahmed, 2006) there is no study demonstrated the role of mother or father separately in diapause induction. According to the recent study 
Diapause Induction In Cotesia Plutellae: Who's More Caring About Their Progeny Fate Mothers ..

long day parents produce a few percentages of diapausing individuals, whereas parents experienced short day produce high diapause incidence in their progeny (Table 1). The results of reciprocal crosses indicated that the diapause incidence in the progeny is under the control of the mother. The incidence of diapause larvae produced by each female whether it was crosses with different male exposed to different condition or male experienced the same condition was the same as diapause incidence produced by the female crossed with male experienced same photoperiodic condition. Diapause in this parasitoid is most likely to be controlled by genetic background of the female. Fathers had no role in determine the developmental fate of his progeny as there was no difference in progeny diapause incidence if each female mated with male experienced long or short photoperiod. In Trichogramma embryophagum diapause induction was only under mother control (Reznik et al. 2011b).

\section{References}

[1] Ahmed, U. A/M.; Shi, Z. H.; Guo, Y.; Zou, X.; Hao. Z.; Pang, S., 2006. Maternal photoperiod effect on and Geographic variation of diapause incidence in Cotesia plutellae (Hymenoptera: Braconidae) from China. Journal of Applied Entomology and Zoology.

[2] Alvi, S.; M.; Momoi, S., 1994. Environmental regulation and geographic adaptation of diapause in Cotesia plutellae (Hymenoptera: Braconidae), a parasitoid of the diamondback moth larvae. Applied Entomology and Zoology 29(1): 89-95.

[3] Anderson, J. F.;Kaya, H. K., 1974. Diapause induction by photoperiod and temperature in the elm spanworm egg parasitoid, Ooencyrtus sp. Annals of the Entomological Society of America 67: 845-849.

[4] Ho, D.H. and Burggren, W.W. 2010. Epigenetics and Transgenerational Transfer: A Physiological Perspective. The Journal of Experimental Biology, 213: 3-16.

[5] MCwatters, H. G.; Saunders, D. S., 1996. The influence of each parents and geographic origin on larval diapause in the blow fly, Calliphora vicina. Journal of insect physiology 42(8): 721-726.

[6] Milonas, P. G.; Savopoulou-Soultani, M., 2000. Diapause induction and termination in the parasitoid Colpoclypeus florus (Hymenoptera: Eulophidae): role of photoperiod and temperature. Annals of the Entomological Society of America 93: 512-518.

[7] Mousseau, T. A.; Dingle, H., 1991. Maternal effects in insect life histories. Annual Review of Entomology 36: 511- 534.

[8] Mousseau, T. A; Fox, C. W., 1998. The adaptive significance of maternal effects. Trends in Ecology and Evolution 13:403-407.

[9] Jason E., Podrabsky. J.E, Garrett I. D, Kohl Z. F. 2010. Alternative developmental pathways associated with diapause regulated by temperature and maternal influences in embryos of the annual killifish Austrofundulus limnaeus Journal of Experimental Biology 213: $3280-3288$

[10] Olof Leimar , John M. McNamara (2015) The Evolution of Transgenerational Integration of Information in Heterogeneous Environments., The American Naturalist 185: 55-69.

[11] Reznik, S.Ya., Vaghina, N.P. and Voinovich, N.D. 2011a. Maternal Influence on Diapause Induction in Trichogramma (Hymenoptera, Trichogrammatidae): The Dynamics of Photosensitivity. Journal of Applied Entomology, 135: 438-445.

[12] Reznik, S.Ya., Voinovich, N.D. and Vaghina, N.P. 2011b. Maternal Regulation of Trichogramma embryophagum Htg. (Hymenoptera: Trichogrammatidae) Diapause: Photoperiodic Sensitivity of Adult Females. Biological Control, 57: 158-162. [CrossRef], [Web of Science $\left.{ }^{\circledR}\right]$

[13] SAS, Institute, 2000. SAS User's Guide: Statistics. Cary, North Carolina.

[14] Saunders, D.S. 2010. Photoperiodism in Insects: Migration and Diapause Responses". In Photoperiodism: The Biological Calendar, Edited by: Nelson, R.J., Denlinger, D.L. and Somers, D.E. 218-257. New York: Oxford University Press

[15] Tachibana S-I; Numata, H., 2004. Parental and direct effects of photoperiod and temperature on the induction of larval diapause in the blow fly Lucilia sericata. Physiological Entomology 29: 39-44.

[16] Wang, X. G.; Liu, S. S.; Guo, S. J.; Lin, W. C., 1999. Effects of host stages and temperature on population parameters of Oomyzus sokolowskii, a larval-pupal parasitoid of Plutellae xylostella. BioControl 44:391-402

[17] Yamaguchi H. and Nakamurak k. 2015. Effects of environmental factors on the regulation of egg diapause in the walking-stick insect, Ramulus irregulariterdentatus (Phasmatodea: Phasmatidae) European Journal of Entomology $112: 35-40$ 